Article

\title{
Comparative Effects of High-Intensity Interval Training vs Moderate-Intensity Continuous Training in Phase III of a Tennis-Based Cardiac Rehabilitation Program: A Pilot Randomized Controlled Trial
}

\author{
Santos Villafaina ${ }^{1}$, María José Giménez-Guervós Pérez ${ }^{2}$ and Juan Pedro Fuentes-García ${ }^{2, *(1)}$ \\ 1 Physical Activity and Quality of Life Research Group (AFYCAV), Faculty of Sport Science, \\ University of Extremadura, 10003 Cáceres, Spain; svillafaina@unex.es \\ 2 Faculty of Sport Science, University of Extremadura, Avda, Universidad S/N, 10003 Cáceres, Spain; \\ mgimenezx@alumnos.unex.es \\ * Correspondence: jpfuent@unex.es; Tel.: +34-92725-7460
}

Received: 27 April 2020; Accepted: 16 May 2020; Published: 19 May 2020

\begin{abstract}
The aim of the present study was to investigate the effects of two tennis-based cardiac rehabilitation programs using two protocols: high-intensity interval training (HIIT) and moderate-intensity continuous training (MICT) in the body composition, physical fitness, heart rate variability, and health-related quality of life. It was performed as a single-blinded randomized controlled trial of 21 people with acute coronary syndrome. The 12-week cardiac rehabilitation program consisted of three sessions per week of 60 min with the main part consisting of 10 sets of $15 \mathrm{~s}$ with $15 \mathrm{~s}$ of active recovery at $85 \%-90 \%$ of their maximum heart rate or $40 \mathrm{~min}$ at $70 \%-85 \%$ of their maximum heart rate in the HIIT and the MICT groups, respectively. Results show that both cardiac rehabilitation programs improved the agility and the mental components of the health-related quality of life ( $p$-value $<0.05$ ). The HIIT protocol also showed a significant improvement in the flexibility of upper and lower limbs ( $p$-value $<0.05)$. Moreover, significant group $\times$ time interaction was found in the handgrip strength in favor of the HIIT group ( $p$-value $=0.035)$. Nevertheless, no significant effects on cardiorespiratory fitness or heart rate variability were found. Importantly, no adverse event was detected, so HIIT appears to be a safe and effective alternative for the rehabilitation of patients with acute coronary syndrome.
\end{abstract}

Keywords: heart rate variability; health-related quality of life; tennis; HIIT; MICT; body composition; flexibility; strength; 6-MWT

\section{Introduction}

Cardiovascular disease (CVD) is one of the most important causes of disability and premature death worldwide [1,2]. It is predicted that in 2030 CVD will contribute to over 23 million deaths annually [3]. In this regard, the coronary artery diseases (CAD) is the leading cause of death and morbidity in Europe [4], with acute coronary syndrome (ACS) being the most common clinical manifestation of CAD [5,6].

Cardiac rehabilitation (CR) is an effective tool for the prevention and management of CVD [7] since the main aim is to improve the overall functional capacity and health-related quality of life (HRQoL) [8,9]. The CR programs have different phases that assist patients from hospitals (phase-I) with the transition to their daily activities through phases II, III, and IV. In this regard, previous studies have shown that CR improves HRQoL [10], body composition [11-13], physical fitness [14,15], or heart rate variability (HRV) $[16,17]$. HRV is a non-invasive index that evaluates autonomic nervous system 
activity (the balance between sympathetic and parasympathetic nervous systems). It is based on successive heartbeat variation over an interval of time [18]. Interestingly, low HRV is associated with an increased risk of death from several causes $[19,20]$.

Despite all these interesting health improvements, less than 3\% of survivors of any CVD enroll in CR programs, and adherence is relatively poor among patients who do enroll in CR settings [21]. Thus, different innovations, combining patient safety, efficacy, and enjoyment have been introduced in CR programs, such as different sport modalities or intensities. Regarding physical exercise modalities, a previous study investigated the effects of tennis-based CR on physical function compared with a traditional cycle-ergometer CR program [22]. In this regard, tennis could be adapted to fulfill all the recommendations for this population [22,23]. In addition, different studies have investigated the introduction of high-intensity interval training (HIIT) in patients with CAD [24]. Results showed that it is a safe, effective, and enjoyable training for patients with CAD [25]. Moreover, when HIIT and moderate-intensity continuous training (MICT) have been compared, greater improvements in aerobic findings have been observed in HIIT protocols [26-28].

However, to the best of our knowledge, the effects of a HIIT tennis-based CR program in phase III have not been studied. Thus, this study aimed to investigate the effects of two tennis-based CR programs using two protocols, HIIT and MICT, on physical fitness, body composition, HRV, and HRQol.

\section{Materials and Methods}

\subsection{Trial Design}

This study was conceived as a single-blinded, randomized controlled trial where participants were randomly allocated into two groups: a tennis-based high-intensity interval training (HIIT) and a tennis-based moderate-intensity continuous training (MICT). All procedures were approved by the University research ethics committee (approval number: 148/2015).

\subsection{Participants}

The intervention was carried out in the Faculty of Sport Sciences (Cáceres, Spain) from February 2016 to April 2016. A total of 21 ACS patients, with a mean age of 55.85 (5.69) years old, were included in this pilot randomized controlled trial. All of them were recruited until 20 January, 2016. The participant inclusion criteria were as follows:

a. Had previously completed phase II of the cardiac rehabilitation program.

b. Men or women with ACS categorized as low risk by a cardiologist [29,30].

c. Had read, accepted, and signed the written informed consent.

Moreover, participants were excluded if the following applied to them:

a. Had a condition that would make the high-intensity exercises contraindicated, such as retinopathy, musculoskeletal injuries, or major balance problems.

A technician randomly allocated the participants into the two groups (HIIT or MICT) using random numbers. The researcher who allocated the participants in the groups did not take part in the evaluation or the data acquisition. Another researcher blinded to the grouping allocation developed the evaluation and data analyses. Participants were unable to be allocated since the two protocols were explained in the informed consent.

\subsection{Intervention}

The HIIT and the MICT training were based on tennis. In this regard, the interventions were performed in an indoor tennis court. The HIIT and MICT programs consisted lasted 12 weeks, with three days per week (32 sessions of $60 \mathrm{~min}$ ) of tennis-based training. The characteristics of these programs were as follows: 
- $\quad$ HIIT: The training started with 8 min of warm-up. For the first $5 \mathrm{~min}$, participants perform joint mobility, walk, or run at $50 \%$ HRmax. Then, three sets of three repetitions (each repetition lasted $10 \mathrm{~s}$ at $80 \% \mathrm{HRmax}$ ) with one minute of active recovery at $50 \% \mathrm{HRmax}$. After $5 \mathrm{~min}$ of active recovery, 10 sets of $15 \mathrm{~s}$ with $15 \mathrm{~s}$ of active recovery at $85 \%-90 \%$ HRmax were performed. The calm-down consisted of $3 \mathrm{~min}$ of active (walking around the tennis court) and $7 \mathrm{~min}$ of passive recovery. All the exercises performed under the HIIT approach were based on tennis.

- $\quad$ MICT: The training started with $8 \mathrm{~min}$ of warm-up. For the first $5 \mathrm{~min}$, participants have to perform joint mobility, walk, or run at 50\%HRmax. Then, participants have to perform $40 \mathrm{~min}$ of tennis-based exercises. Since tennis is an intermittent sport, following the recommendation of Casasola [23] and García, Giraldo, Barrado, and Casasola [22], some adaptations were performed to maintain the intensity at $70 \%-85 \% \mathrm{HRmax}$ during the session. For example, participants were allowed to let the ball bounce twice or using mainly doubles rather than singles games. Moreover, four different on-court movement intensities were introduced (i.e., walking slowly, walking fast, jogging, and running), to maintain the effort within the heart rate limits. Lastly, the calm-down consisted of $3 \mathrm{~min}$ of active (walking around the tennis court) and $7 \mathrm{~min}$ of passive recovery.

All the training sessions were conducted and supervised by a kinesiologist with six years of tennis training experience. The heart rate of participants during the sessions was monitored using a Polar RS800CX (Finland) heart rate monitor.

\subsection{Outcomes}

\subsubsection{Physical Fitness and Body Composition}

Bimanual Handgrip. Handgrip strength was measured using a hand dynamometer (Takei TKK 5401 Digital Handgrip Dynamometer, Tokyo Japan). Participants had to squeeze the dynamometer with an optimal grip-span. The sum of the better of two attempts for each hand was used in the analyses.

Broad Jump Test. Lower limb strength was assessed by the broad jump test. Participants were instructed to bend their knees (the depth of the flexion was self-selected) and bring their arms behind their bodies. Then, they have to extend their legs, move their arms forward, and jump as far as possible. The distance, in centimeters, of the best of two attempts were used to the statistical analyses [31].

T-test. Four cones were set up in a "T" shape. Cone A and B were placed $9.14 \mathrm{~m}$ apart from each other. Cone $\mathrm{B}$ and $\mathrm{C}$, and $\mathrm{B}$ and $\mathrm{D}$ were placed $4.57 \mathrm{~m}$ apart from each other. The participant started at cone $\mathrm{A}$, touched the base of cone $\mathrm{B}$ with their right hand, and then had to move left and shuffle sideways to cone C. Once they had touched its base (this time with their left hand), participants had to shuffle sideways to the right to cone $\mathrm{D}$ (touching its base with the right hand). Then, they shuffle back to cone B, touching it with the left hand, and run backwards to cone A. The time that participants employed in this agility test was used in the analyses [32].

Back Scratch Test. This test was used to assess upper limb flexibility [33]. Participants had to try to touch with the middle finger of both hands behind the back. Thus, they had to pass one hand behind their head, flexing their elbow and directing the middle finger down while they make the opposite movement with the other hand. After that, they performed the movements changing the right and left arm actions. A staff member assessed the distance between the fingers with a measuring tape [31].

Sit and Reach Test. Participants had to sit on the floor and place their legs fully extended and directing the tip of the foot towards the ceiling. At the evaluator's signal, the subjects had to flex their hips trying to touch the tip of their big toe with the middle finger of their hand. The result was negative if the hands did not reach the foot, and positive if it was passed [34]. The best result of two attempts was used in the analyses [31].

6-Minute Walking Test (6-MWT). This test was used to measure cardiorespiratory fitness, determining the maximum distance (in meters) the patients can walk in 6 min following a rectangular 
path of $45.7 \mathrm{~m}$ [35]. The following well-established equation has been used to estimates the maximum oxygen uptake $\left(\mathrm{VO}_{2 \max }\right)[36]$ :

$$
\text { Mean Peak } \mathrm{VO}_{2}=4.948+0.023 \times \text { mean 6MWD (meters) } \text {. }
$$

Both the $6 \mathrm{MWD}$ and the $\mathrm{VO}_{2 \max }(\mathrm{mL} / \mathrm{kg} / \mathrm{min})$ estimation were included in the analyses.

\subsubsection{Heart Rate Variability}

A Polar RS800CX (Finland) heart rate monitor was used to record HRV, following the recommendations of the Task Force of the European Society of Cardiology and the North American Society of Pacing and Electrophysiology [37]. Therefore, a short-term record (5 $\mathrm{min}$ ) was employed for each HRV acquisition. HRV data was extracted using the Kubios HRV software (v. 3.3) [38,39]. A middle filter was applied to correct possible artifacts, identifying those RR intervals, which are shorter/longer than $0.25 \mathrm{~s}$, compared to the previous beats average. Correction replaces the identified artifacts by cubic spline interpolation.

Time-domain, frequency-domain, and non-linear variables were analyzed in the present study. Regarding time domain, mean heart rate (mean HR), the standard deviation of the successive NN intervals (SDNN); RR50 count divided by the total number of all RR ranges (Pnn50), and the square root of differences between adjacent RR intervals (RMSSD). In the frequency domain, the low frequency (LF, 0.04-0.15 Hz) and high frequency (HF, 0.15-0.4 Hz) ratio (LF/HF) were studied. Lastly, in the non-linear measures, the RR variability from heartbeat to short term Poincaré graph (width) (SD1), the RR variability from heartbeat to long-term Poincaré graph (length) (SD2) and the Sample Entropy (SampEn) were included.

\subsubsection{Quality of Life}

SF-12v2. The SF-12 is a multipurpose short-form to assess the health-related quality of life (HRQoL) with only 12 questions, all selected from the SF-36 Health Survey [40]. This questionnaire evaluates eight dimensions: physical functioning (PF), role physical (RP), bodily pain (BP), general health $(\mathrm{GH})$, vitality (VT), social functioning (SF), role emotional (RE), and mental health (MH). Moreover, two summary scores are estimated: physical summary score (PCS) and mental summary score (MSC). The scores represent the person's health status $(100=$ full HRQoL, $0=$ death $)$ [41].

\subsection{Statistical Analysis}

The SPSS statistical package (version 20.0; SPSS, Inc., Chicago, Ill.) was used to analyze the data. According to the results of the Shapiro-Wilk and Kolmogorov-Smirnov tests, non-parametric analyses were conducted.

Data from all 21 initial participants were used to conduct the intention-to-treat analysis by multiple imputations (MI) of missing values following the Sterne, et al. [42] guidelines. Our missing data were classified as missing at random. The SPSS software was used to perform MI of data.

Mann-Whitney $U$ test was employed to study the differences at baseline in the age, weight, body mass index (BMI), 6MWT, SDNN, MCS, and PCS.

The difference between post and pre was calculated for each variable. These differences were used to study the effects of the intervention between groups by the Mann-Whitney $U$ test in the different variables. Within-group effects were explored using the Wilcoxon signed-rank test (HIIT and MICT separately).

Additionally, effect sizes were calculated for the non-parametric tests through $\mathrm{r}$, which is classified as follows: 0.5 is a large effect, 0.3 is a medium effect, and 0.1 is a small effect [43,44]. The alpha level of significance (0.05) was adjusted according to the Benjamini-Hochberg procedure to avoid type I error derived from multiple comparisons [45]. 


\section{Results}

Figure 1 shows the flow diagram of the participants. A total of 27 people with ACS were assessed for eligibility. However, six declined to participate in the study. Therefore, 21 adults with ACS were randomized into two groups: HIIT $(n=11)$ and MICT $(n=10)$. One participant of the MICT group did not complete the intervention. In this regard, intent-to-treat analyses were applied to this participant's data. No cardiovascular-related adverse events were reported in relation to HIIT or MICT sessions.

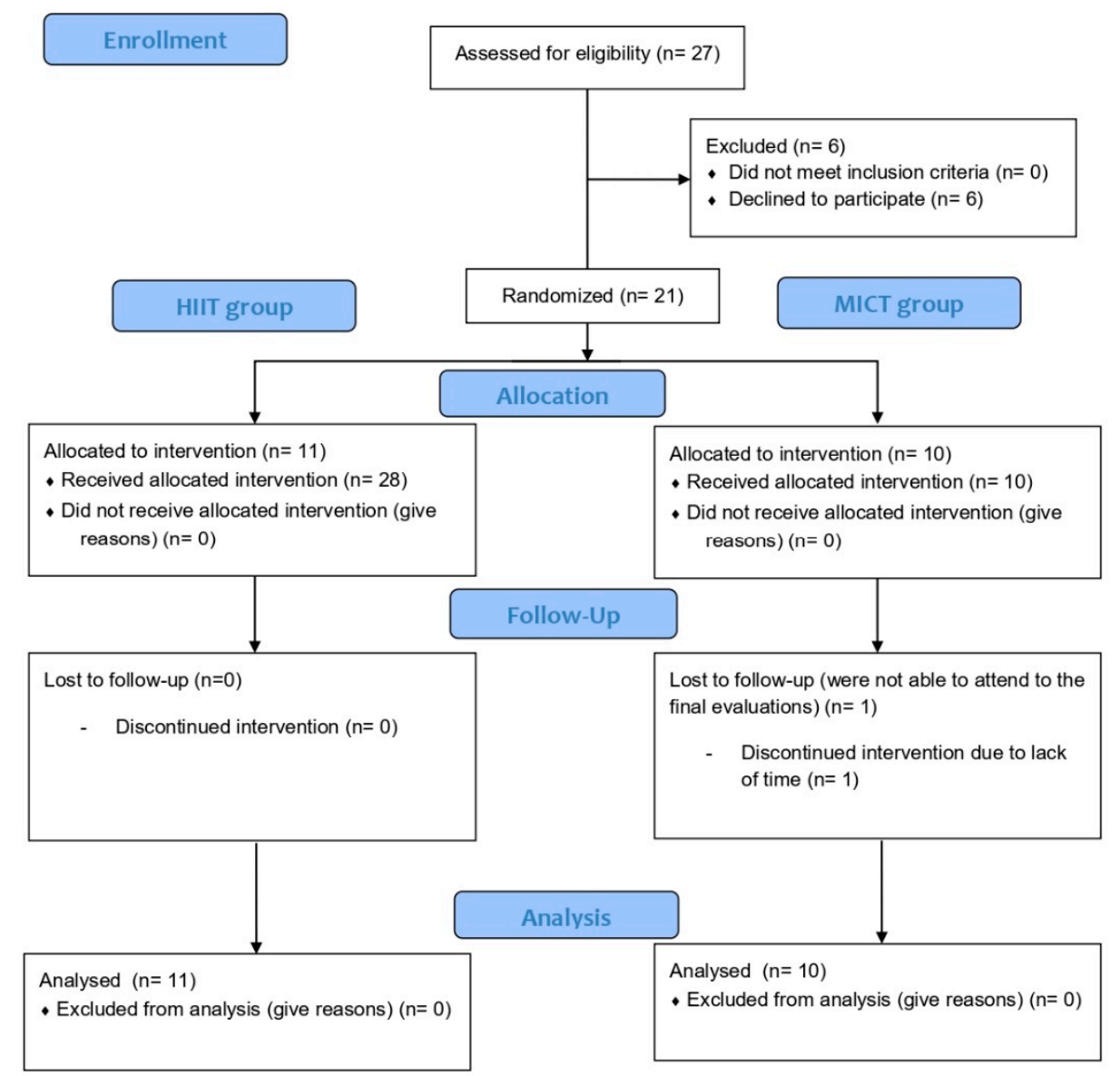

Figure 1. Flow chart of participants.

Table 1 shows the characteristics of the participants included in the study. A total of 21 people with ACS, with a mean age of 55.85 (5.69) years old, participated in the study. Significant differences were not observed ( $p$-value $>0.05$ ) between HIIT and MICT groups in weight, BMI, 6-MWT, SDNN, MCS, or PCS.

Table 1. Baseline characteristics of the participants.

\begin{tabular}{lcccc}
\hline & All (n = 21) & $\begin{array}{c}\text { HIIT Group } \\
(\mathbf{n}=\mathbf{1 1})\end{array}$ & $\begin{array}{c}\text { MICT Group } \\
(\mathbf{n}=\mathbf{1 0})\end{array}$ & $p$-value * \\
\hline Age (years) & $55.85(5.69)$ & $55.27(7.13)$ & $56.55(3.50)$ & 0.148 \\
Weight $(\mathrm{kg})$ & $86.67(13.68)$ & $87.87(16.64)$ & $85.34(10.23)$ & 0.751 \\
BMI $\left(\mathrm{kg} / \mathrm{m}^{2}\right)$ & $30.54(4.22)$ & $31.04(5.14)$ & $29.98(3.11)$ & 0.438 \\
6-MWT $(\mathrm{m})$ & $583.26(72.40)$ & $584.09(82.71)$ & $603.54(83.62)$ & 0.218 \\
SDNN & $40.88(19.19)$ & $50.89(22.71)$ & $40.18(21.66)$ & 0.425 \\
MCS & $35.24(5.64)$ & $35.91(6.56)$ & $35.01(4.73)$ & 0.573 \\
PCS & $35.48(5.64)$ & $35.99(7.53)$ & $34.41(2.42)$ & 0.888 \\
\hline
\end{tabular}

BMI: Body Mass Index; MICT: Moderate-intensity continuous training; HIIT: High-Intensity interval training; PCS: Physical components summary; MCS: Mental components summary; kg: kilograms; m: meters; cm: centimeters; s: seconds; * The $p$-values were calculated with U Mann-Whitney test. 
Table 2 shows the effect of HIIT and MICT in the body composition and physical fitness of people with ACS. Within-group analyses show that the performance in the T-test (agility) improved in both HIIT ( $p$-value $=0.03$ ) and MICT ( $p$-value $=0.047)$ groups with an effect size that can be classified as large. Moreover, the HIIT group had significantly improved performance in the back scratch ( $p$-value $=0.011)$ and sit and reach ( $p$-value $=0.008)$ flexibility tests, with an effect size that can be classified as large. In addition, between-group analyses showed a significant group*time interaction in the bimanual handgrip ( $p$-value $=0.035$ ) with greater benefits observed in the HIIT group.

Table 2. Intent-to-treat of 16-weeks of HIIT and MICT in the body composition and physical fitness of people with acute coronary syndrome (ACS).

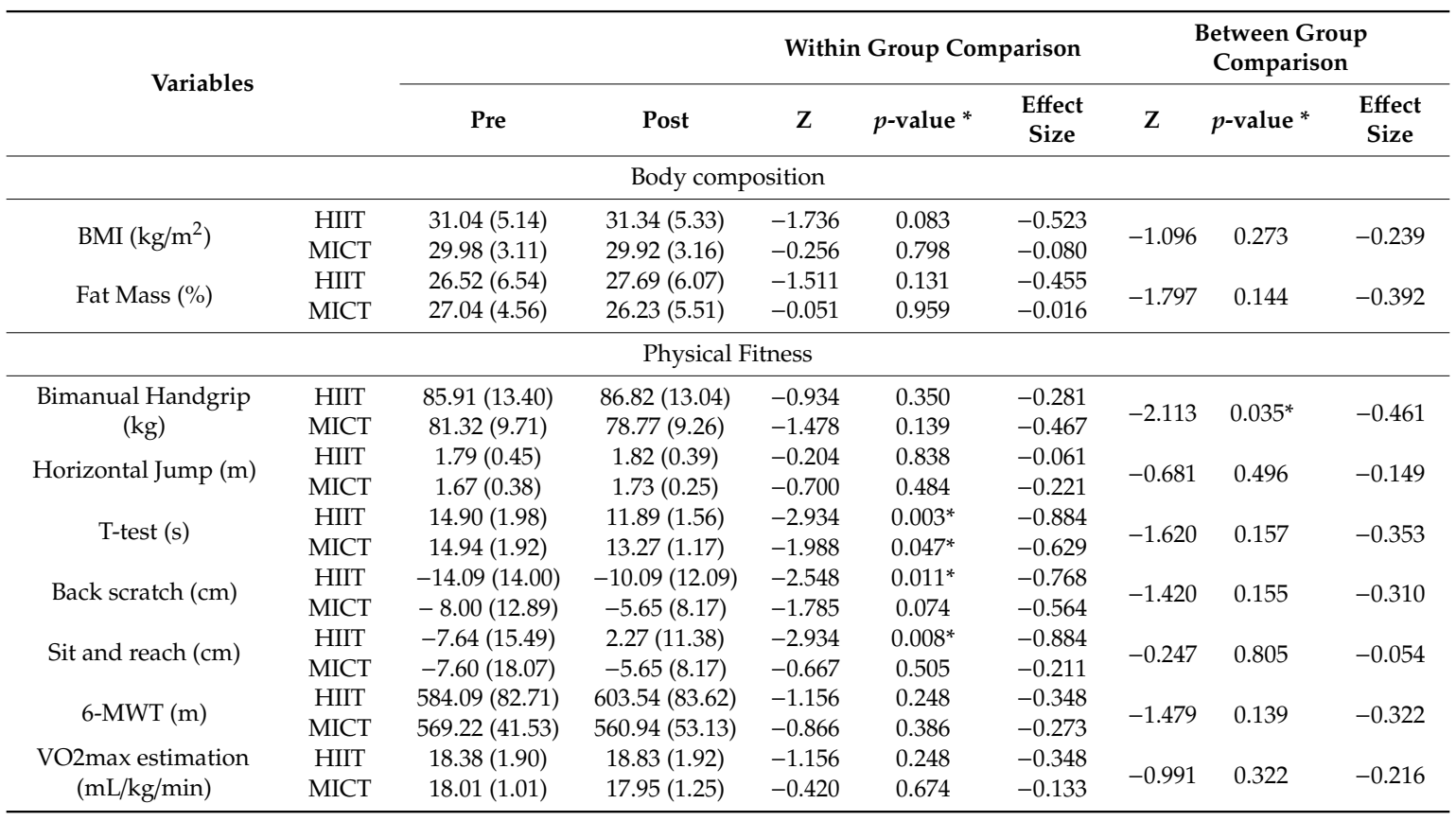

* $p$-value < 0.05; 6-MWT: Six-minutes walking test; BMI: Body mass index; HIIT: High-Intensity interval training; MICT: Moderate-intensity continuous training; $\mathrm{VO}_{2 \max }$ : maximum oxygen uptake.

Table 3 shows the effects of HIIT and MICT on HRV. Neither within- nor between-group differences were observed for time-domain, frequency-domain, or non-linear measures after 12 weeks of intervention.

Table 3. Intent-to-treat of 16-weeks of HIIT and MICT in the heart rate variability of people with ACS.

\begin{tabular}{|c|c|c|c|c|c|c|c|c|c|}
\hline \multicolumn{2}{|c|}{ HRV Measures } & Pre & Post & \multicolumn{3}{|c|}{ Within Group Comparison } & \multicolumn{3}{|c|}{ Between Group Comparison } \\
\hline $\begin{array}{l}\text { Heart Rate } \\
\text { (bpm) }\end{array}$ & MICT & $67.55(13.60)$ & $67.84(11.18)$ & -0.255 & 0.799 & -0.081 & -0.563 & 0.573 & -0.123 \\
\hline SDNN & HIIT & $50.89(22.71)$ & 43.87 (19.75) & -0.978 & 0.328 & -0.295 & -0.423 & 0.673 & -0.092 \\
\hline RMSSD & MICT & $25.74(18.38)$ & $25.30(25.90)$ & -1.478 & 0.139 & -0.467 & -0.810 & 0.418 & -0.177 \\
\hline \multirow{2}{*}{ Pnn50 } & HIIT & $12.34(16.01)$ & $13.40(19.50)$ & -0.178 & 0.859 & -0.054 & \multirow{2}{*}{-0.493} & \multirow{2}{*}{0.622} & \multirow{2}{*}{-0.108} \\
\hline & MICT & $6.93(13.22)$ & $7.63(15.31)$ & -1.423 & 0.155 & -0.450 & & & \\
\hline \multirow{2}{*}{$\mathrm{HF}$} & HIIT & $28.00(15.11)$ & $36.06(20.19)$ & -1.334 & 0.182 & -0.402 & \multirow{2}{*}{-1.972} & \multirow{2}{*}{0.100} & \multirow{2}{*}{-0.430} \\
\hline & MICT & $28.60(18.22)$ & $22.78(11.03)$ & -1.326 & 0.185 & -0.419 & & & \\
\hline
\end{tabular}


Table 3. Cont.

\begin{tabular}{|c|c|c|c|c|c|c|c|c|c|}
\hline \multirow{2}{*}{\multicolumn{2}{|c|}{ HRV Measures }} & \multirow[b]{2}{*}{ Pre } & \multirow[b]{2}{*}{ Post } & \multicolumn{3}{|c|}{ Within Group Comparison } & \multicolumn{3}{|c|}{ Between Group Comparison } \\
\hline & & & & $\mathbf{Z}$ & $p$-value * & $\begin{array}{l}\text { Effect } \\
\text { Size }\end{array}$ & $\mathbf{Z}$ & $p$-value * & $\begin{array}{l}\text { Effect } \\
\text { Size }\end{array}$ \\
\hline \multirow{2}{*}{ LF/HF } & HIIT & $3.58(2.32)$ & $3.32(3.97)$ & -0.800 & 0.424 & -0.241 & \multirow{2}{*}{-1.408} & \multirow{2}{*}{0.159} & \multirow{2}{*}{-0.307} \\
\hline & MICT & $4.24(4.13)$ & $6.51(9.66)$ & -0.968 & 0.333 & -0.306 & & & \\
\hline \multirow{2}{*}{ SD1 } & HIIT & 24.19 (16.69) & $24.37(15.30)$ & -0.178 & 0.859 & -0.054 & \multirow{2}{*}{-0.986} & \multirow[b]{2}{*}{0.324} & \multirow[b]{2}{*}{-0.215} \\
\hline & MICT & $18.22(13.00)$ & 17.48 (17.75) & -1.478 & 0.139 & -0.467 & & & \\
\hline \multirow{2}{*}{ SD2 } & HIIT & $67.25(28.82)$ & $56.27(25.18)$ & -1.245 & 0.213 & -0.375 & \multirow{2}{*}{-0.352} & \multirow{2}{*}{0.725} & \multirow{2}{*}{-0.077} \\
\hline & MICT & $52.97(29.06)$ & $52.86(23.77)$ & -0.764 & 0.445 & -0.242 & & & \\
\hline \multirow{2}{*}{ SampEn } & HIIT & $1.46(0.19)$ & $1.45(0.34)$ & -0.178 & 0.859 & -0.054 & \multirow{2}{*}{-0.986} & \multirow{2}{*}{0.324} & \multirow{2}{*}{-0.215} \\
\hline & MICT & $1.32(0.22)$ & $1.18(0.27)$ & -1.784 & 0.074 & -0.564 & & & \\
\hline
\end{tabular}

${ }^{*} p$-value < 0.05; HIIT: High-Intensity interval training; MICT: Moderate-intensity continuous training.

Table 4 shows the effects of HIIT and MICT in HRQoL of people with ACS. Significant within-group improvements $(p$-value $<0.05)$ in both HIIT and MICT groups were observed in PCS and MH. Moreover, both groups significantly reduced PCS ( $p$-value $<0.05)$. A significant reduction in BP was also observed in the HIIT group. However, a significant improvement in VT was observed in the HIIT group. Significant group $\times$ time interactions were not found in HRQoL between HIIT and MICT.

Table 4. Intent-to-treat of 16-weeks of HIIT and MICT in HRQoL of people with ACS.

\begin{tabular}{|c|c|c|c|c|c|c|c|c|c|}
\hline \multirow{2}{*}{\multicolumn{2}{|c|}{ Quality of Life }} & \multirow[b]{2}{*}{ Pre } & \multirow[b]{2}{*}{ Post } & \multicolumn{3}{|c|}{ Within Group Comparison } & \multicolumn{3}{|c|}{ Between Group Comparison } \\
\hline & & & & $\mathbf{Z}$ & $p$-value * & $\begin{array}{c}\text { Effect } \\
\text { Size }\end{array}$ & $\mathbf{Z}$ & $p$-value * & $\begin{array}{c}\text { Effect } \\
\text { Size }\end{array}$ \\
\hline \multicolumn{10}{|c|}{ Summary Scores } \\
\hline \multirow{2}{*}{ MCS } & HIIT & $35.99(7.53)$ & $54.47(3.38)$ & -2.934 & $0.003 *$ & -0.884 & \multirow{2}{*}{-0.070} & \multirow{2}{*}{0.944} & \multirow{2}{*}{-0.015} \\
\hline & MICT & $34.41(2.42)$ & $53.44(4.78)$ & -2.803 & $0.005 *$ & -0.886 & & & \\
\hline \multirow{2}{*}{ PCS } & HIIT & $35.91(6.56)$ & $28.47(3.90)$ & -2.934 & $0.003 *$ & -0.884 & \multirow{2}{*}{-0.282} & \multirow{2}{*}{0.778} & \multirow{2}{*}{-0.061} \\
\hline & MICT & $35.01(4.73)$ & $27.77(3.70)$ & -2.599 & $0.009 *$ & -0.822 & & & \\
\hline \multicolumn{10}{|c|}{ SF-12v2 domains } \\
\hline \multirow{2}{*}{ PF } & HIIT & $22.73(28.40)$ & $13.64(17.19)$ & -1.081 & 0.279 & -0.326 & \multirow{2}{*}{-1.120} & \multirow{2}{*}{0.263} & \multirow{2}{*}{-0.244} \\
\hline & MICT & $20.00(32.91)$ & $16.67(25.00)$ & -0.447 & 0.655 & -0.141 & & & \\
\hline \multirow{2}{*}{$\mathrm{RP}$} & HIIT & $70.45(14.00)$ & $81.82(23.29)$ & -1.172 & 0.241 & -0.353 & \multirow{2}{*}{0.349} & \multirow{2}{*}{0.727} & \multirow{2}{*}{0.076} \\
\hline & MICT & $65.00(19.36)$ & 73.61 (19.21) & -1.421 & 0.155 & -0.449 & & & \\
\hline \multirow{2}{*}{ BP } & HIIT & $18.18(25.23)$ & $2.27(7.54)$ & -2.121 & $0.034 *$ & -0.639 & \multirow{2}{*}{-0.676} & \multirow{2}{*}{0.499} & \multirow{2}{*}{-0.147} \\
\hline & MICT & $15.00(26.87)$ & $5.56(11.02)$ & -1.633 & 0.102 & -0.516 & & & \\
\hline \multirow{2}{*}{ GH } & HIIT & $52.73(19.28)$ & 64.09 (20.59) & -1.769 & 0.077 & -0.533 & \multirow{2}{*}{-0.301} & \multirow{2}{*}{0.763} & \multirow{2}{*}{-0.006} \\
\hline & MICT & $50.50(20.88)$ & 58.89 (24.59) & -1.852 & 0.064 & -0.585 & & & \\
\hline \multirow{2}{*}{ VF } & HIIT & 31.82 (29.77) & $75.00(15.81)$ & -2.595 & $0.009 *$ & -0.782 & & & \\
\hline & MICT & $37.50(21.24)$ & 63.89 (18.16) & -1.852 & 0.064 & -0.585 & -1.184 & 0.237 & 58 \\
\hline & HIIT & $29.55(33.20)$ & $6.82(16.17)$ & -1.638 & 0.101 & -0.494 & & & \\
\hline $\mathrm{SF}$ & MICT & $20.00(22.97)$ & $11.11(22.05)$ & -1.300 & 0.194 & -0.411 & 15 & 0.398 & \\
\hline & HIIT & 78.41 (19.44) & 79.54 (20.37) & -0.322 & 0.748 & -0.010 & & & \\
\hline RE & MICT & $75.00(22.82)$ & $86.11(17.05)$ & -1.469 & 0.142 & -0.464 & & 0.312 & \\
\hline & HIIT & 19.32 (15.17) & $86.36(11.80)$ & -2.952 & $0.003 *$ & -0.890 & & & \\
\hline MH & MICT & $15.00(19.36)$ & 79.17 (21.65) & -2.533 & 0.011 * & -0.801 & & 0.817 & 50 \\
\hline
\end{tabular}

* $p$-value e< 0.05; BP: Bodily Pain; GH: General Health; HIIT: High-Intensity interval training; MCS: Mental components summary; MH: Mental Health; MICT: Moderate-intensity continuous training; PCS: Physical components summary; PF: Physical functioning; RE: Role emotional; RP: Role Physical; SF: Social Functioning; VT: Vitality.

\section{Discussion}

The present study investigated the effects of two tennis-based CR programs focused on HIIT and MICT protocols. Results showed that both CR programs improved the agility and the mental components of HRQoL. The HIIT protocol also showed an improvement in flexibility. Moreover, significant group*time interaction was found in the handgrip strength in favor of the HIIT group. 
Previous studies have shown improvements in physical fitness after CR programs $[14,15]$. Our results are consistent with these results, showing agility enhancements in both HIIT and MICT groups. Moreover, participants of the HIIT group also improved their flexibility in both upper and lower limbs. These results are relevant since a decline in flexibility or agility causes difficulties in performing daily activities [46]. Moreover, flexibility has a great impact on HRQoL, but it is usually under-evaluated in randomized controlled trials [47]. In addition, our results showed a significant group*time interaction in the handgrip strength in favor of the HIIT group. This is quite relevant since grip strength is correlated with mortality [48] and could predict cardiac adverse events in patients with cardiac disorders [49]. Despite this interesting finding, HIIT appears to provide similar benefits to MICT for improving body composition or cardiorespiratory fitness, which is also in line with previous research [50].

HRV is a non-invasive index that evaluates the balance between sympathetic and parasympathetic nervous systems. Moreover, $\mathrm{HRV}$ is associated with increased risk of death from several causes [19], and is an interesting tool in the management of chronic diseases, such as diabetes [51] or CAD [52]. In this regard, our results did not show any significant effect of HIIT or MICT protocols, or any significant group interaction between these programs. Nevertheless, previous studies have shown controversial results in this topic. Whereas some studies reported positive effects of HIIT or MICT protocols on the HRV of people with CAD [16], others did not find significant effects in this outcome [53-55]. In this regard, considering both our results and the previous research, it can be hypothesized that interventions should be longer than 4 months to improve HRV. This hypothesis is also supported by previous studies in other populations [51,56,57].

Our results showed an increase in the mental component of HRQoL in both HIIT and MICT protocols. This is consistent with previous studies were CR programs have been useful tools to improve HRQoL of people with CAD $[10,58,59]$. Moreover, within-group differences indicated that vitality significantly increased in the HIIT group. This is relevant since previous studies have shown that HIIT protocols could have a greater impact on HRQoL than MICT protocols in people with CAD [60,61]. However, a significant decline in the physical component of HRQoL was detected in both HIIT and MICT. This could be influenced by the results of the pain dimension, where the within-group analyses indicated that pain was increased in the HIIT group (also a tendency can be found in the MICT). Although hypothetically, these results might be influenced by delayed onset muscle soreness, which participants had not experienced before the intervention.

Adherence is one of the greater challenges of CR programs. In this regard, previous studies have pointed out that the lack of motivation could be the reason why CAD patients dropped out of CR programs [62,63]. However, HIIT protocols have shown to be a safe, effective, and enjoyable tool for patients with CAD within CR [25]. This is quite relevant since adherence is maintained only when participation is perceived as enjoyable [64]. Moreover, our CR program is based on tennis, which addresses elements of the self-determination theory [65]. These could be the reasons why only one participant interrupted the intervention, achieving 100\% and $90 \%$ adherence in HIIT and MICT protocols, respectively.

This study has some limitations that should be acknowledged. Firstly, the small sample size could mean that only greater differences would reach the significance level. Secondly, only men participated in this study, so results cannot be extrapolated to women with ACS.

\section{Conclusions}

Results showed that both MICT and HIIT protocols improved the agility and mental components of HRQoL. Moreover, the HIIT group showed an improvement in flexibility and vitality dimensions of HRQoL. This is relevant since a decline in flexibility or agility causes difficulties in performing daily activities. In addition, the main finding of the study was the significant group $\times$ time interaction found in the handgrip strength in favor of the HIIT group. This is quite important due to the relationship 
between handgrip strength and mortality. Importantly, any adverse event was detected, so HIIT appears to be a safe and effective alternative for the rehabilitation of patients with ACS.

Author Contributions: Conceptualization, S.V. and J.P.F.-G.; data curation, S.V. and M.J.G.-G.P.; formal analysis, S.V.; funding acquisition, J.P.F.-G.; investigation, S.V. and M.J.G.-G.P.; methodology, S.V., M.J.G.-G.P., and J.P.F.-G.; project administration, J.P.F.-G.; resources, J.P.F.-G.; software, S.V.; supervision, S.V., M.J.G.-G.P., and J.P.F.-G.; validation, M.J.G.-G.P. and J.P.F.-G.; visualization, M.J.G.-G.P. and J.P.F.-G.; writing-original draft, S.V.; Writing-review and editing, M.J.G.-G.P. and J.P.F.-G. All authors have read and agreed to the published version of the manuscript.

Funding: This study was completed thanks to the contribution of the Ministry of Economy and Infrastructure of the Junta de Extremadura through the European Regional Development Fund. A way to make Europe. (GR18129). The author SV was also supported by a grant from the regional department of economy and infrastructure of the Government of Extremadura and the European Social Fund (PD16008).

Conflicts of Interest: The authors declare no conflict of interest.

\section{References}

1. Roth, G.A.; Abate, D.; Abate, K.H.; Abay, S.M.; Abbafati, C.; Abbasi, N.; Abbastabar, H.; Abd-Allah, F.; Abdela, J.; Abdelalim, A. Global, regional, and national age-sex-specific mortality for 282 causes of death in 195 countries and territories, 1980-2017: A systematic analysis for the Global Burden of Disease Study 2017. Lancet 2018, 392, 1736-1788. [CrossRef]

2. Roth, G.A.; Johnson, C.; Abajobir, A.; Abd-Allah, F.; Abera, S.F.; Abyu, G.; Ahmed, M.; Aksut, B.; Alam, T.; Alam, K. Global, regional, and national burden of cardiovascular diseases for 10 causes, 1990 to 2015. J. Am. Coll. Cardiol. 2017, 70, 1-25. [CrossRef] [PubMed]

3. Mozaffarian, D.; Benjamin, E.J.; Go, A.S.; Arnett, D.K.; Blaha, M.J.; Cushman, M.; Das, S.R.; De Ferranti, S.; Després, J.-P.; Fullerton, H.J. Executive summary: Heart disease and stroke statistics-2016 update: A report from the American Heart Association. Circulation 2016, 133, 447-454. [CrossRef] [PubMed]

4. Dégano, I.R.; Elosua, R.; Kaski, J.C.; Fernández-Bergés, D.J.; Grau, M.; Marrugat, J. Estabilidad de la placa aterosclerótica y la paradoja del sur de Europa. Rev. Española Cardiol. 2013, 66, 56-62. [CrossRef]

5. Cequier, Á. El registro MASCARA desenmascara la realidad asistencial del manejo de los síndromes coronarios agudos en España. Rev. Española Cardiol. (Engl. Ed.) 2008, 61, 793-796. [CrossRef]

6. Dégano, I.R.; Elosua, R.; Marrugat, J. Epidemiology of acute coronary syndromes in Spain: Estimation of the number of cases and trends from 2005 to 2049. Rev. Española Cardiol. (Engl. Ed.) 2013, 66, 472-481. [CrossRef]

7. Selig, S.E.; Levinger, I.; Williams, A.D.; Smart, N.; Holland, D.J.; Maiorana, A.; Green, D.J.; Hare, D.L. Exercise $\&$ Sports Science Australia Position Statement on exercise training and chronic heart failure. J. Sci. Med. Sport 2010, 13, 288-294.

8. Thompson, D.R.; Yu, C.-M. Quality of life in patients with coronary heart disease-I: Assessment tools. Health Qual. Life Outcomes 2003, 1, 42. [CrossRef]

9. Piepoli, M.F.; Corra, U.; Dendale, P.; Frederix, I.; Prescott, E.; Schmid, J.P.; Cupples, M.; Deaton, C.; Doherty, P.; Giannuzzi, P.; et al. Challenges in secondary prevention after acute myocardial infarction: A call for action. Eur. J. Prev. Cardiol. 2016, 23, 1994-2006. [CrossRef]

10. Francis, T.; Kabboul, N.; Rac, V.; Mitsakakis, N.; Pechlivanoglou, P.; Bielecki, J.; Alter, D.; Krahn, M. The effect of cardiac rehabilitation on health-related quality of life in patients with coronary artery disease: A meta-analysis. Can. J. Cardiol. 2019, 35, 352-364. [CrossRef]

11. Lear, S.A.; Spinelli, J.J.; Linden, W.; Brozic, A.; Kiess, M.; Frohlich, J.J.; Ignaszewski, A. The Extensive Lifestyle Management Intervention (ELMI) after cardiac rehabilitation: A 4-year randomized controlled trial. Am. Heart J. 2006, 152, 333-339. [CrossRef] [PubMed]

12. Giannuzzi, P.; Temporelli, P.L.; Marchioli, R.; Maggioni, A.P.; Balestroni, G.; Ceci, V.; Chieffo, C.; Gattone, M.; Griffo, R.; Schweiger, C. Global secondary prevention strategies to limit event recurrence after myocardial infarction: Results of the GOSPEL study, a multicenter, randomized controlled trial from the Italian Cardiac Rehabilitation Network. Arch. Intern. Med. 2008, 168, 2194-2204. [CrossRef] [PubMed] 
13. Pedersen, L.R.; Olsen, R.H.; Anholm, C.; Astrup, A.; Eugen-Olsen, J.; Fenger, M.; Simonsen, L.; Walzem, R.L.; Haugaard, S.B.; Prescott, E. Effects of 1 year of exercise training versus combined exercise training and weight loss on body composition, low-grade inflammation and lipids in overweight patients with coronary artery disease: A randomized trial. Cardiovasc. Diabetol. 2019, 18, 127. [CrossRef] [PubMed]

14. Molino-Lova, R.; Pasquini, G.; Vannetti, F.; Paperini, A.; Forconi, T.; Polcaro, P.; Zipoli, R.; Cecchi, F.; Macchi, C. Effects of a structured physical activity intervention on measures of physical performance in frail elderly patients after cardiac rehabilitation: A pilot study with 1-year follow-up. Intern. Emerg. Med. 2013, 8, 581-589. [CrossRef]

15. O'Neill, D.; Forman, D.E. The importance of physical function as a clinical outcome: Assessment and enhancement. Clin. Cardiol. 2020, 43, 108-117. [CrossRef]

16. Munk, P.S.; Butt, N.; Larsen, A.I. High-intensity interval exercise training improves heart rate variability in patients following percutaneous coronary intervention for angina pectoris. Int. J. Cardiol. 2010, 145, 312-314. [CrossRef]

17. Fiogbe, E.; Ferreira, R.; Sindorf, M.A.G.; Tavares, S.A.; de Souza, K.P.; de Castro Cesar, M.; Lopes, C.R.; Moreno, M.A. Water exercise in coronary artery disease patients, effects on heart rate variability, and body composition: A randomized controlled trial. Physiother. Res. Int. J. Res. Clin. Phys. Ther. 2018, 23, e1713. [CrossRef]

18. Thayer, J.F.; Ahs, F.; Fredrikson, M.; Sollers, J.J., III; Wager, T.D. A meta-analysis of heart rate variability and neuroimaging studies: Implications for heart rate variability as a marker of stress and health. Neurosci. Biobehav. Rev. 2012, 36, 747-756. [CrossRef]

19. Dekker, J.M.; Crow, R.S.; Folsom, A.R.; Hannan, P.J.; Liao, D.; Swenne, C.A.; Schouten, E.G. Low heart rate variability in a 2-minute rhythm strip predicts risk of coronary heart disease and mortality from several causes-The ARIC study. Circulation 2000, 102, 1239-1244. [CrossRef]

20. Fang, S.C.; Wu, Y.L.; Tsai, P.S. Heart Rate Variability and Risk of All-Cause Death and Cardiovascular Events in Patients with Cardiovascular Disease: A Meta-Analysis of Cohort Studies. Biol. Res. Nurs. 2020, 22, 45-56. [CrossRef]

21. Piepoli, M.F.; Corra, U.; Benzer, W.; Bjarnason-Wehrens, B.; Dendale, P.; Gaita, D.; McGee, H.; Mendes, M.; Niebauer, J.; Zwisler, A.-D.O. Secondary prevention through cardiac rehabilitation: From knowledge to implementation. A position paper from the Cardiac Rehabilitation Section of the European Association of Cardiovascular Prevention and Rehabilitation. Eur. J. Cardiovasc. Prev. Rehabil. 2010, 17, 1-17. [CrossRef] [PubMed]

22. García, J.P.F.; Giraldo, V.M.A.; Barrado, J.J.G.; Casasola, C.D. Tennis training sessions as a rehabilitation instrument for patients after acute myocardial infarction. J. Sports Sci. Med. 2013, 12, 316. [PubMed]

23. Casasola, C.D. Analysis of heart rate during a tennis training session and its relationship with heart-healthy index. J. Sport Health Res. 2010, 2, 26-34.

24. Ribeiro, P.A.B.; Boidin, M.; Juneau, M.; Nigam, A.; Gayda, M. High-intensity interval training in patients with coronary heart disease: Prescription models and perspectives. Ann. Phys. Rehabil. Med. 2017, 60, 50-57. [CrossRef]

25. Keech, A.; Holgate, K.; Fildes, J.; Indraratna, P.; Cummins, L.; Lewis, C.; Yu, J. High-intensity interval training for patients with coronary artery disease: Finding the optimal balance. Int. J. Cardiol. 2020, 298, 8-14. [CrossRef]

26. Liou, K.; Ho, S.; Fildes, J.; Ooi, S.-Y. High intensity interval versus moderate intensity continuous training in patients with coronary artery disease: A meta-analysis of physiological and clinical parameters. Heart Lung Circ. 2016, 25, 166-174. [CrossRef]

27. Hannan, A.L.; Hing, W.; Simas, V.; Climstein, M.; Coombes, J.S.; Jayasinghe, R.; Byrnes, J.; Furness, J. High-intensity interval training versus moderate-intensity continuous training within cardiac rehabilitation: A systematic review and meta-analysis. Open Access J. Sports Med. 2018, 9, 1-17. [CrossRef]

28. Xie, B.; Yan, X.; Cai, X.; Li, J. Effects of high-intensity interval training on aerobic capacity in cardiac patients: A systematic review with meta-analysis. BioMed Res. Int. 2017, 2017, 5420840. [CrossRef] 
29. Roffi, M.; Patrono, C.; Collet, J.-P.; Mueller, C.; Valgimigli, M.; Andreotti, F.; Bax, J.J.; Borger, M.A.; Brotons, C.; Chew, D.P. 2015 ESC Guidelines for the management of acute coronary syndromes in patients presenting without persistent ST-segment elevation: Task Force for the Management of Acute Coronary Syndromes in Patients Presenting without Persistent ST-Segment Elevation of the European Society of Cardiology (ESC). Eur. Heart J. 2016, 37, 267-315.

30. Yan, A.T.; Yan, R.T.; Tan, M.; Casanova, A.; Labinaz, M.; Sridhar, K.; Fitchett, D.H.; Langer, A.; Goodman, S.G. Risk scores for risk stratification in acute coronary syndromes: Useful but simpler is not necessarily better. Eur. Heart J. 2007, 28, 1072-1078. [CrossRef]

31. ACSM. ACSM's Guidelines for Exercise Testing and Prescription; Wolters Kluwer: Alfen, The Netherlands, 2017.

32. Pauole, K.; Madole, K.; Garhammer, J.; Lacourse, M.; Rozenek, R. Reliability and validity of the T-test as a measure of agility, leg power, and leg speed in college-aged men and women. J. Strength Cond. Res. 2000, 14, 443-450.

33. Phillips, W.T. Senior fitness test manual. J. Aging Phys. Act. 2002, 10, 110-111.

34. Rikli, R.E.; Jones, C.J. Development and validation of a functional fitness test for community-residing older adults. J. Aging Phys. Act. 1999, 7, 129-161. [CrossRef]

35. Hanson, L.C.; McBurney, H.; Taylor, N.F. The retest reliability of the six-minute walk test in patients referred to a cardiac rehabilitation programme. Physiother. Res. Int. 2012, 17, 55-61. [CrossRef] [PubMed]

36. Ross, R.M.; Murthy, J.N.; Wollak, I.D.; Jackson, A.S. The six minute walk test accurately estimates mean peak oxygen uptake. BMC Pulm. Med. 2010, 10, 31. [CrossRef]

37. Camm, A.J.; Malik, M.; Bigger, J.T.; Breithardt, G.; Cerutti, S.; Cohen, R.J.; Coumel, P.; Fallen, E.L.; Kennedy, H.L.; Kleiger, R.E. Heart rate variability: Standards of measurement, physiological interpretation and clinical use. Task Force of the European Society of Cardiology and the North American Society of Pacing and Electrophysiology. Ann. Noninvasive Electrocardiol. 1996, 1, 151-181.

38. Da Costa de Rezende Barbosa, M.P.; da Silva, N.T.; de Azevedo, F.M.; Pastre, C.M.; Marques Vanderlei, L.C. Comparison of Polar ((R)) RS800G3 heart rate monitor with Polar ((R)) S810i and electrocardiogram to obtain the series of RR intervals and analysis of heart rate variability at rest. Clin. Physiol. Funct. Imaging 2016, 36, 112-117. [CrossRef]

39. Tarvainen, M.P.; Niskanen, J.-P.; Lipponen, J.A.; Ranta-Aho, P.O.; Karjalainen, P.A. Kubios HRV-heart rate variability analysis software. Comput. Methods Programs Biomed. 2014, 113, 210-220. [CrossRef]

40. Ware, J., Jr.; Kosinski, M.; Keller, S.D. A 12-Item Short-Form Health Survey: Construction of scales and preliminary tests of reliability and validity. Med. Care 1996, 34, 220-233. [CrossRef]

41. Ware, J., Jr.; Kosinski, M.; Turner-Bowker, D.J.R.I.Q.I. How to Score Version 2 of the SF-12®Health Survey (with a Supplement Documenting Version 1); Lincoln: Dearborn, MI, USA, 2002.

42. Sterne, J.A.; White, I.R.; Carlin, J.B.; Spratt, M.; Royston, P.; Kenward, M.G.; Wood, A.M.; Carpenter, J.R. Multiple imputation for missing data in epidemiological and clinical research: Potential and pitfalls. BMJ (Clin. Res. Ed.) 2009, 338, b2393. [CrossRef]

43. Fritz, C.O.; Morris, P.E.; Richler, J.J. Effect Size Estimates: Current Use, Calculations, and Interpretation. J. Exp. Psychol. Gen. 2012, 141, 2-18. [CrossRef] [PubMed]

44. Coolican, H. Research Methods and Statistics in Psychology; Psychology Press: Oxford, UK, 2017.

45. Benjamini, Y.; Hochberg, Y. Controlling the false discovery rate: A practical and powerful approach to multiple testing. J. R. Stat. Soc. Ser. B (Methodol.) 1995, 289-300. [CrossRef]

46. Tuna, H.D.; Edeer, A.O.; Malkoc, M.; Aksakoglu, G. Effect of age and physical activity level on functional fitness in older adults. Eur. Rev. Aging Phys. Act. 2009, 6, 99. [CrossRef]

47. Busch, A.J.; Schachter, C.L.; Overend, T.J.; Peloso, P.M.; Barber, K.A. Exercise for fibromyalgia: A systematic review. J. Rheumatol. 2008, 35, 1130-1144. [PubMed]

48. Buckner, S.L.; Dankel, S.J.; Bell, Z.W.; Abe, T.; Loenneke, J.P. The Association of Handgrip Strength and Mortality: What Does It Tell Us and What Can We Do with It? Rejuvenation Res. 2019, 22, 230-234. [CrossRef] [PubMed]

49. Pavasini, R.; Serenelli, M.; Celis-Morales, C.A.; Gray, S.R.; Izawa, K.P.; Watanabe, S.; Colin-Ramirez, E.; Castillo-Martínez, L.; Izumiya, Y.; Hanatani, S. Grip strength predicts cardiac adverse events in patients with cardiac disorders: An individual patient pooled meta-analysis. Heart 2019, 105, 834-841. [CrossRef] 
50. LiQiang, S.; JinMei, F.; ShunLi, S.; GuangGao, Z.; Wei, C.; ChuanChuan, D.; MingHui, Q. Effects of HIIT and MICT on cardiovascular risk factors in adults with overweight and/or obesity: A meta-analysis. PLoS ONE 2019, 14, e0210644.

51. Villafaina, S.; Collado-Mateo, D.; Fuentes, J.P.; Merellano-Navarro, E.; Gusi, N. Physical Exercise Improves Heart Rate Variability in Patients with Type 2 Diabetes: A Systematic Review. Curr. Diabetes Rep. 2017, 17, 110. [CrossRef]

52. Hernando, D.; Kähönen, M.; Lázaro, J.; Lehtinen, R.; Nieminen, T.; Nikus, K.; Lehtimäki, T.; Bailón, R.; Viik, J. Coronary artery disease diagnosis by means of heart rate variability analysis using respiratory information. In EMBEC E NBC 2017; Springer: Berlin, Germany, 2017; pp. 270-273.

53. Badrov, M.B.; Wood, K.N.; Lalande, S.; Sawicki, C.P.; Borrell, L.J.; Barron, C.C.; Vording, J.L.; Fleischhauer, A.; Suskin, N.; McGowan, C.L. Effects of 6 Months of Exercise-Based Cardiac Rehabilitation on Autonomic Function and Neuro-Cardiovascular Stress Reactivity in Coronary Artery Disease Patients. J. Am. Heart Assoc. 2019, 8, e012257. [CrossRef]

54. Sandercock, G.R.H.; Hodges, L.D.; Das, S.K.; Brodie, D.A. The impact of short term supervised and home-based walking programmes on heart rate variability in patients with peripheral arterial disease. J. Sports Sci. Med. 2007, 6, 471-476.

55. Currie, K.D.; Rosen, L.M.; Millar, P.J.; McKelvie, R.S.; MacDonald, M.J. Heart rate recovery and heart rate variability are unchanged in patients with coronary artery disease following 12 weeks of high-intensity interval and moderate-intensity endurance exercise training. Appl. Physiol. Nutr. Metab. 2013, 38, 644-650. [CrossRef] [PubMed]

56. Hottenrott, K.; Hoos, O.; Esperer, H.D. Heart rate variability and physical exercise. Current status. Herz 2006, 31, 544-552. [CrossRef] [PubMed]

57. Villafaina, S.; Collado-Mateo, D.; Domínguez-Muñoz, F.J.; Gusi, N.; Fuentes-Garcia, J.P. Effects of exergames on heart rate variability of women with fibromyalgia: A randomized controlled trial. Sci. Rep. 2020, 10, 5168. [CrossRef] [PubMed]

58. de la Cuerda, R.C.; Diego, I.M.A.; Martín, J.J.A.; Sánchez, A.M.; Page, J.C.M. Cardiac rehabilitation programs and health-related quality of life. State of the art. Rev. Española Cardiol. (Engl. Ed.) 2012, 65, 72-79. [CrossRef]

59. Seki, E.; Watanabe, Y.; Sunayama, S.; Iwama, Y.; Shimada, K.; Kawakami, K.; Sato, M.; Sato, H.; Mokuno, H.; Daida, H. Effects of phase III cardiac rehabilitation programs on health-related quality of life in elderly patients with coronary artery disease. Circ. J. 2003, 67, 73-77. [CrossRef]

60. Villelabeitia Jaureguizar, K.; Vicente-Campos, D.; Ruiz Bautista, L.; Hernandez de la Pena, C.; Arriaza Gómez, M.J.; Calero Rueda, M.J.; Fernandez Mahillo, I. Effect of high-intensity interval versus continuous exercise training on functional capacity and quality of life in patients with coronary artery disease. J. Cardiopulm. Rehabil. Prev. 2016, 36, 96-105. [CrossRef]

61. Guiraud, T.; Nigam, A.; Gremeaux, V.; Meyer, P.; Juneau, M.; Bosquet, L. High-intensity interval training in cardiac rehabilitation. Sports Med. 2012, 42, 587-605. [CrossRef]

62. Smith, J.; Garton-Smith, J.; Briffa, T.; Maiorana, A. The development of a new cardiac rehabilitation needs assessment tool (CRNAT) for individualised secondary prevention. Heart Lung Circ. 2015, 24, 458-464. [CrossRef]

63. Reid, R.D.; Morrin, L.I.; Higginson, L.A.J.; Wielgosz, A.; Blanchard, C.; Beaton, L.J.; Nelson, C.; McDonnell, L.; Oldridge, N.; Wells, G.A. Motivational counselling for physical activity in patients with coronary artery disease not participating in cardiac rehabilitation. Eur. J. Prev. Cardiol. 2012, 19, 161-166. [CrossRef]

64. Rhodes, R.E.; Fiala, B.; Conner, M. A review and meta-analysis of affective judgments and physical activity in adult populations. Ann. Behav. Med. 2009, 38, 180-204. [CrossRef]

65. Ryan, R.M.; Williams, G.C.; Patrick, H.; Deci, E.L. Self-determination theory and physical activity: The dynamics of motivation in development and wellness. Hell. J. Psychol. 2009, 6, 107-124.

(C) 2020 by the authors. Licensee MDPI, Basel, Switzerland. This article is an open access article distributed under the terms and conditions of the Creative Commons Attribution (CC BY) license (http://creativecommons.org/licenses/by/4.0/). 